
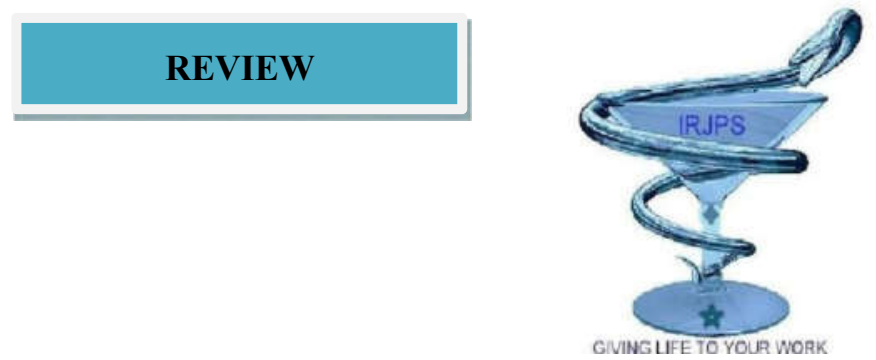

\title{
“A REVIEW ON NANOPARTICLES: THEIR PREPARATION METHOD AND APPLICATIONS"
}

\section{Beena Kumari}

Department of Pharmaceutical Education and Research, Bhagat Phool Singh Mahila Vishwidylaya, South campus, Bhainswal Kalan, Sonepat, Haryana, India.

\section{Submitted on: 30.04.18; $\quad$ Revised on: 12.05.18; Accepted on: 27.05.18}

\begin{abstract}
Nanoparticles are the simplest form of structures with sizes in the nm range. In principle any collection of atoms bonded together with a structural radius of $<100 \mathrm{~nm}$ can be considered a nanoparticle. In the present time nanoparticles are widely used in many dosage form due to their good solubilty, less size and better penetrability. Nanoparticles can be prepared by using the various methods such as Emulsion-Solvent Evaporation Method, Double Emulsion and Evaporation Method, Salting Out Method, Emulsions Diffusion Method, Solvent Displacement/Precipitation method, Polymerization method and Coacervation or ionic gelation method. Applications of nanoparticles in micro wiring are cell specific, internalization, vaccine delivery and gene delivery. Nanoparticles are used in the field of medicines also for the treatment of cancer or for the orthopaedic implants. Nanoparticles shows high solubility and fast penetration that's why they are used in almost formulation now a days.
\end{abstract}

KEYWORDS: Gold Nanoparticles (AuNPs), Magnetic Resonance Imaging (MRI), Polyvinylpyrrolidone (PVP), Blood brain barrier (BBB).

Corresponding author: Beena Kumari E-mail: beenasangwan051119993@gmail.com Mobile: +91- 9992185034
Indian Research Journal of Pharmacy and Science; 17(2018)1420-1426; Journal Home Page: https://www.irjps.in DOI: 10.21276/irjps.2018.5.2.3 


\section{INTRODUCTION:}

Nanotechnology is getting developed at various levels such as materials, systems and devices. At present in commercial applications and scientific information, the most innovativelevel is nanomaterials. In nanotechnology, a minor object that acts as a complete unit in terms ofits properties and transport is known as a particle. It can be categorizedaccording to sizes as fine particle and ultrafine particle. In terms of diameter, fine particles are sized between 100 and 2500 nanometers, while ultrafine particles cover a rangebetween 1 and 100 nanometers. Nanoparticlesare also sized between 1 and 100 nanometers similar to the ultrafine particles. Nanoparticles may or may not demonstrate sizerelated properties that varyknowingly from those observed in bulk materials and fine particles. ${ }^{1}$ Thus nanoparticles are sized less than a few $100 \mathrm{~nm}$. This reduction in size brings about significant changes in their physical properties with respect to those observed in bulk materials. They can be mineral, metallic, polymer-based or a combination of materials. Due to a wide variety of potential applications in optical,electronic fields and biomedical, Nanoparticle research is currently an area of intense scientific interest. The reason behindnanoparticles are attractive is based on their unique and important features, such as their surface to mass ratio, which is much larger than that of other particles and materials, their ability to adsorb and carry other compounds such as drugs, probes and proteinsas well as permitting thecatalytic promotion of reactions. ${ }^{2}$

Advantages ${ }^{3,4}$

- After parenteral administration toachieve both passive and active drug targeting particle size and surface characteristics of nanoparticles can be easily manipulated.

- To achieve high drug therapeutic efficacy and less side effects, during the transportation they control and sustain release of the drug and at the site oflocalization, altering distribution of the drug and subsequent clearance of the drug.

- By attaching targeting ligands to surface ofparticles or use of magnetic guidance site-specific targeting can be achieved.

- Includingoral, intra-ocular,parenteral and nasal,the system can be used for various routes of administration.

- Within the body,drug delivery to tiny areas can be achieved better by nanoparticles.

- Engineering enables researchers to exercise precisely on this scale and previously control over the biomaterials and physical features of polymers.
- Nanoparticles provide efficient delivery of drug to various parts of the body by overcoming the resistance offered by the physiological barriers in the body which is directly affected by particle size.

- Nanoparticles can aid in efficient drug delivery by improving aqueous solubility of poorly soluble drugs andincrease bioavailability for organizedrelease of drug molecules, and accurate drugtargeting.

- For targeted drug delivery, the surface properties of nanoparticles can be altered forproteins, small molecules, peptides, and nucleicacids loaded nanoparticles are not recognized byimmune system and targeted to particular tissue types efficiently.

- By targetingnano drug carriersdrug toxicity can be reduced and more efficient drug distribution can be provided.

- Over various anatomic extremities of bodysuch as blood brain barrier (BBB) nano carriers holds potential to deliver biotech drugs.

\section{Nanoparticles: Types}

Silver: These are proved to be most effective because of their good antimicrobial efficacy against bacteria,viruses and other eukaryotic microorganisms. ${ }^{5,6}$ Among all the nanomaterials they are most widely used as antimicrobial agents, for sunscreen lotions, water treatment and in textile industries etc. ${ }^{7,8} \mathrm{By}$ using the plants i.e.Capsicum annuum $^{9}$, Azadirachta indica ${ }^{10}$ and Carica papaya $^{11}$ the successful biosynthesis of silver nanoparticles have been reported.

Gold: For identification of protein interactions in immunochemical studies gold nanoparticles (AuNPs) are used. In DNA fingerprinting they are used as lab tracer to detect existence of DNA in a sample. Aminoglycoside antibiotics i.e.streptomycin, gentamycin and neomycin are also detected by using these nanoparticles. Detection of cancer stem cells, diagnosis of cancer and identification of different classes of bacteriadone by using Gold nano rods. ${ }^{12,13}$

Alloy: From the bulk samples, structural properties of alloy nanoparticles are different. ${ }^{14}$ Silver flakes are most commonly used due totheir highestelectrical conductivity among other metal fillers, their oxides also have relatively greater conductivity. ${ }^{15}$ By both metals and over ordinary metallic NPs bimetallic alloy nanoparticles properties are influenced whichshow moreadvantages. ${ }^{16}$ 
Magnetic: Magnetic nanoparticles are known to be biocompatible i.e.maghemite and magnetite. For magnetic resonance imaging (MRI), guided drug delivery, targeted cancer treatment (magnetic hyperthermia), gene therapy, stem cell sorting and manipulation and for DNA analysis they have been actively considered. ${ }^{17}$

\section{Preparation of nanoparticles}

For the preparation of nanoparticles, the selection of the appropriate method is based on the drug to be loaded and physicochemical properties of the polymer. The primary preparation methods of nanoparticles includes:

\section{Emulsion-Solvent Evaporation Method}

The nanoparticles are mostly prepared by using this method.Two steps are mainly involved in this method. In an aqueous phase, emulsification of the polymer solution required in the first step. While in the second step, evaporation of polymer solutionoccurs and nanospheres are formed by inducing the polymer precipitation. Collection of nanoparticles is done by ultracentrifugation and to remove free drug or residue, washed with distilled water and for storage these are lyophilized. ${ }^{18}$ This method is also known as solvent evaporation methodand highpressure emulsification. ${ }^{19}$ This technique involves homogenization under high pressure and overall stirring to remove organic solvent. ${ }^{20}$ By adjusting the stirring rate, viscosity of organic and aqueous phases, temperature, type and amount of dispersing agent the size can be controlled. $^{21}$ However to lipid soluble drugs, this technique can be applied and by the scale up issues limitation are imposed. Polymers used are PLA, Poly $(\beta-\text { hydroxybutyrate) (PHB })^{22}$, Poly (caprolactone) (PCL) ${ }^{23}, \mathrm{PLGA}^{24}$, cellulose acetate phthalate ${ }^{25}$, and $\mathrm{EC}^{26}$ in this method.

\section{Double Emulsion and Evaporation Method}

Poor entrapment of hydrophilic drugs is the main drawback of this method.Therefore to encapsulate hydrophilic drug the double emulsion technique is engaged, inwhich aqueous drug solutions is added to organic polymer solution with vigorous stirringto form w/o emulsions. With continuous stirring to form mixed emulsion (w/o/w), this w/o emulsion is added into another aqueous phase. Then by the evaporation solvent is removed and by centrifugation at high speed nano particles can be isolated. Before lyophilisation the prepared nanoparticles must be washed. ${ }^{27}$ The variables used in this method are;incorporated quantity of hydrophilic drug, the amount of polymer, the volume of aqueous phase andthe stabilizer concentration. The characterization of nano particles also affected by these variables. ${ }^{28}$

\section{Salting Out Method}

By using salting-out from aqueous solution the water-miscible solvent is separated using this method. ${ }^{29}$ Initially in a solvent, polymer and drug are dissolved which is consequently containing the saltingout agent (electrolytes, such as calcium chloride and magnesium chloride or sucrose as non- electrolytes) and polyvinylpyrrolidone(PVP) or hydroxyethylcellulose as a colloidal stabilizer into an aqueous gel areemulsified.This oil in water emulsion is diluted with water or with an aqueous phase to increase the diffusion of solvent, which indicates the formation of nanospheres. Several parameters such as electrolyte concentration, concentration of polymers in the organic phase, type of stabilizer, stirring rate, internal/external phase ratio can be varied. ${ }^{25}$ This technique leads to high efficiency and easily scaled up in the preparation of Ethyl cellulose, PLA and Poly( methacrylic) acids nanospheres. ${ }^{30,31}$ Salting out may be useful for heat sensitive substances because an increase of temperature does not require in this technique. An exclusive application to lipophilic drug and the extensive nanoparticles washing steps are the drawbacks of this method.

\section{Emulsions Diffusion Method}

To prepare nanoparticles, emulsions diffusion method is another method which iscommonly used. The encapsulating polymer is dissolvedin a solvent which is partially miscible with water such as propylene carbonate, benzyl alcohol and the initial thermodynamic equilibrium of both liquids saturated with water should be ensured. Subsequently, The polymer-water saturated solvent phase is emulsified in an aqueous solution containing stabilizer, leading to solvent diffusion to the external phase and according to the oil-topolymer ratio nanospheres or nanocapsules are formed. Finally, according to boiling point the solvent is removed by evaporation or filtration. This technique has several advantages, such as high reproducibility (batch-to-batch), no requirement of homogenization, high encapsulation efficiencies (generally 70\%),simplicity, narrow size distribution and ease of scale-up.

But some drawbacks of this method are: the high volumes of water to be eliminated from the suspension and reduced encapsulation efficiencyduring emulsification becausein the saturated-aqueous external phase leakage of watersoluble drug. ${ }^{32}$ Examples of some drug- loaded nano particles which were produced by this technique; cyclosporine (cy-A-); loaded sodium glycolate nanoparticles $^{33}$, mesotetra (hydroxyphenyl) porphyrin-loaded PLGA (p-THPP) nano particles ${ }^{34}$ and nano particles of doxorubicin-loaded PLGA. ${ }^{35}$ 


\section{Solvent Displacement/Precipitation method}

Solvent displacement includes from an organic solution, the precipitation of a preformed polymer and in the aqueous medium the diffusion of the organic solvent in the presence or absence of surfactant. In a semi-polar water miscible solvent such as acetone or ethanol, polymers, drug and lipophilic surfactant are dissolved. Then solution is poured or injected using the magnetic stirring, into stabilizer containing aqueous solution. By the rapid solvent diffusion nano particles are formed. Then under reduced pressure solvent is removed from the suspension. The particles size is also affected by rate of addition of the organic phase into the aqueous phase. It was observed that by increasing the rate of mixing, both particles size and drug entrapment decreases. ${ }^{36}$ For most of the poorly soluble drugs nano precipitation method is well suited. By adjusting preparation parameters; nanosphere size, and drug release can be controlled effectively. While adjusting concentration of polymer results in good production of smaller sized nanospheres. ${ }^{37}$

\section{Polymerization method}

In this method, polymerization of monomers is done in an aqueous solution andafter polymerization completed, drug is incorporated either by adsorption onto the nanoparticles or by being dissolved in the polymerization medium. To remove various stabilizers and surfactants, employed for polymerization by ultra centrifugation the nanoparticle suspension is then purified and in an isotonic surfactant-free medium re-suspending the particles. For making polybutyl cyanoacrylate or poly(alkylcyano acrylate) nanoparticles, this technique has been reported. ${ }^{7}$ Formation of nanocapsule and their particle size affected by the surfactants and stabilizers concentration used. ${ }^{38}$

\section{Coacervation or ionic gelation method}

On the preparation of nanoparticles much research has been focused using biodegradable hydrophilic polymers such as chitosan, sodium alginate and gelatin. A method for preparing hydrophilic chitosan nanoparticles by ionic gelation developed by Calvo and co-workers. ${ }^{39,40}$ The method contains two aqueousphases, in which one is the polymer chitosan and the other phase is a polyanioni.e. sodium tripolyphosphate In this method, interaction of positively charged amino group of chitosan with negative charged tri polyphosphate occurs which form coacervates with an anometer size range. Electrostatic interaction between two aqueous phases results in the formation of coacervates, while ionic interaction conditions at room temperature results in transition from liquid to gel due to ionic gelation.

\section{Application of Nanoparticles}

Nanomedicine has incredible prospects for the improvement of the diagnosis and treatment of human diseases. An environmentally acceptable procedure for the biosynthesis of nanoparticles is the use of microbes. To revolutionize a wide array of tools in biotechnology nanotechnology has potential so that they are more cheaper, personalized, safer, portable and easier to administer.

\section{Timed release of the drug}

To prevent nonspecific toxicity the drug must not diffuse out of the particle while it is still in the circulatory system, and must remain encapsulated until the particle binds to the target.

At the site of disease nanoparticles can be significantly used in targeted drug delivery due to which some major influences occurs such as

1. Drug bioavailability can be improved by using the nanoparticles.

2. Targeting of drugs to a specific site

3. To improve the uptake of poorly soluble drugs

4. Chemotherapeutic agents such as dexamethasone, doxorubicin 5fluorouracil and paclitaxel have been successfully formulated using nanomaterials. $^{41}$

\section{Cell specificity}

Enhancement of cell specificity by conjugating antibodies to carbon nanotubes with fluorescent or radiolabelling. ${ }^{42}$

\section{Internalization}

Internalization within mammalian cells can be achieved by surface- functionalized carbon nanotubes.

\section{Vaccine delivery}

Conjugation with peptides may be used as vaccine delivery structures.

\section{Gene silencing}

ighly selective therapy is required for cancer therapy where tumor cells will be selectively modulated. In this case with small interfering RNA gene silencing has been done. By targeting functionalized single walled carbon nanotubes with siRNA this can be achieved in the targeted cell to silence targeted gene expression. ${ }^{43}$ 


\section{In diagnostics}

It was reported that compounds that are bound to nanotubes enhance the efficiency of diagnostic methods. This property of functionalization and high length to diameter aspect ratio (which provides a high surface to volume ratio), assists in designing the highly efficient biosensors. ${ }^{44}$ Due to very interesting physicochemical properties over other drug delivery and diagnostic systems carbon nanotubes offer diverse advantages. The physicochemical properties are high thermal conductivity, ordered structure with high aspect ratio, ultra-light weight, metallic, high electrical conductivity, high mechanical strength or semimetallic behaviour. $^{45}$

\section{Nanotechnology in Medicine}

\section{Drug Delivery}

In medicine one application of nanoparticle is presently being developed which involves deliver the drugs, heat, light or other elements to specific types of cells in form of nanoparticles (such as cancer cells). Nanoparticles are engineered so that they are drawn to disease cells, which will allow direct treatment of those cells. This technique will not only decrease damage to healthy cells in the body but will enable earlier detection of disease. Another technique to treat cancer cells employs on delivering chemotherapy drugs to cancer cells and applying heat to the cells. ${ }^{46}$ Gold nanorods are attached to DNA strands by researchers which act as a scaffold, holding together with the nanorod and the administered drug. The gold nanorod absorbs the infrared light when infrared light illuminates the cancer tumor, turning it into heat. The chemotherapy drug is released by the heat and it helps to destroy the cancer cells. ${ }^{47}$

\section{Diagnostic Techniques}

To make very early detection of cancer tumors easier, researchers are developing a nanoparticle intended. The nanoparticles release "biomarkers" molecules when the glue to cancer tumors is detected and the biomarkers are known as peptides. The idea is that even at initial stages of cancer, each nanoparticle carries several peptides which results in a high concentration of these biomarkers will, enabling early detection of the disease. The color of the nanorod shifts when proteins accumulate on the nanorods. The test is designed to be done fast and inexpensively for early detection of a problem? .

\section{Anti-Microbial Techniques}

Staph infections can be fought by a nanoparticle cream which contains nitric oxide gas, known to kill bacteria. Studies on mice have shown that using the nanoparticle cream to release nitric oxide gas at the site of staph abscesses significantly reduced the infection. If an infection is started by the harmful bacteria releasing the antibiotics, coating with nanocapsules containing antibiotics, burn dressing will open. Quicker treatment of an infection is done which reduces the number of times a dressing has to be changed. ${ }^{48}$

\section{Use of silver nanoparticles as antibacterial agent}

Ultrasonography uses sound waves to create an image for many different purposes. These sound waves are transmitted through the body and bounce off of tissue and return to a receiver. This receiver measures the time it takes for the sound wave to reflect and return to the place of origin, which is perceived as a distance and is converted into an electrical signal, which is then converted into an image by the computer.

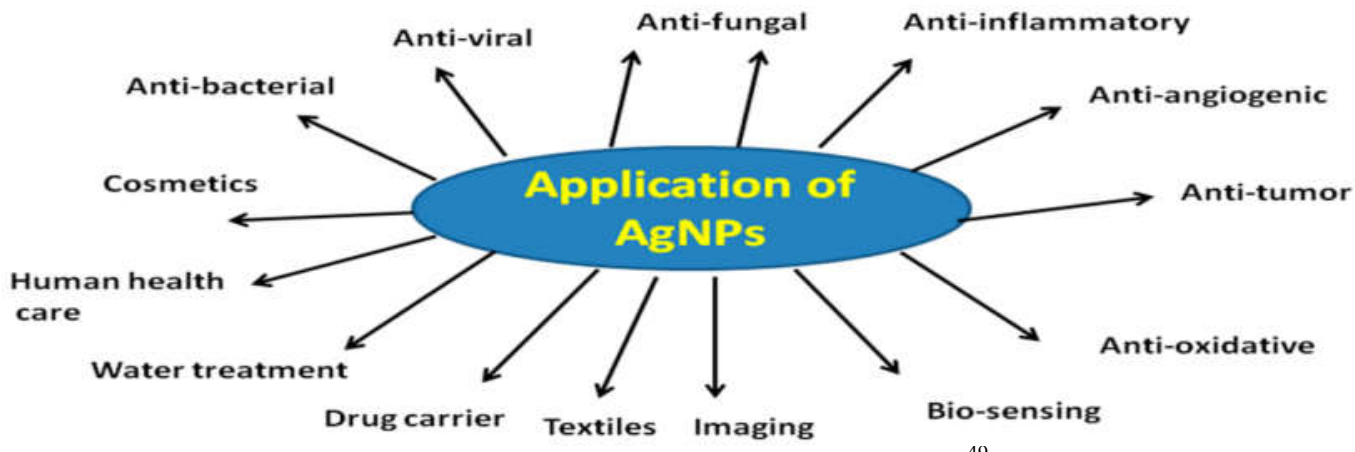

Figure 1: Application of silver nanoparticle ${ }^{49}$

This type of medical imaging is used in many branches of medicine spanning from obstetrics to oncology. Unfortunately, minor details could be missed with ultrasonography because the image may not be of the best quality. ${ }^{49}$
CONCLUSION: Due to their incredible properties, nanoparticles have become significant in many fields in recent years such as energy, healthcare, environment, agriculture etc. Nanoparticle technologies have great potentials, 
being able to convert poorly soluble, poorly absorbed and labile biologically active substance

\section{REFERENCES}

1. Cristina Buzea, Ivan Pacheco, Kevin Robbie., Nanomaterials and Nanoparticles:Sources and Toxicity.,Biointerphases.,2007; 2: MR17MR71.

2. Paul JA, Borm and Wolfgang Kreyling., Toxicological Hazards of Inhaled Nanoparticles- Potential Implications for Drug Delivery., J Nanosci Nanotech., 2004; 4(6):111.

3. Mohsen J, Zahra B., Protein nanoparticle: A unique system as drug delivery vehicles., African Journal of Biotechnology., 2008; 25:4926-4934.

4. Rawat M, Singh D, Saraf S, Nanocarriers: Promising Vehicle for Bioactive Drugs. Biol. Pharm. Bull.2006; 29(9):1790-1798.

5. Gong P, Li H, He X, Wang K, Hu J, Tan W, Tan S, Zhang XY., Preparation and antibacterial activity of $\mathrm{Fe}_{3} \mathrm{O}_{4}$ at $\mathrm{Ag}$ nanoparticles., Nanotech., 2007; 18: 604-611.

6. Mahendra R, Yadav A, Gade A., Biotech Adv., 2009; 27(1): 76-83.

7. Rai M, Yadav A, Gade A., Biotech Adv., 2009; 27(2): 813-817.

8. Sharma VK, Ria AY, Lin Y., Adv Colloid and Interface Sci., 2009; 145: 83-96.

9. Bar H, Bhui DK, Sahoo GP, Sarkar P, De SP, Misra A., Colloids and Surfaces., Physicochem. Eng. Aspects., 2009; 339: 134-139.

10. Shankar SS, Rai A, Ankamwar B, Singh A, Ahmad A, Sastry., Biological synthesis of triangular gold nanoprisms., Nat Mater., 2004; 3: $482-488$

11. Jha AK, Prasad K., Green Synthesis of Silver Nanoparticles Using Cycas Leaf. Int J Green Nanotech: Physics and Chemistry., 2010; 1: 110-117.

12. Baban D, Seymour LW., Control of tumour vascular permeability., Adv Drug Deliv Rev., 1998; 34: 109-119.

13. Tomar A, Garg G., Short Review on Application of Gold Nanoparticles., Global Journal of Pharmacology., 2013; 7 (1): 34-38.

14. Ceylan A, Jastrzembski K, Shah SI., Enhanced solubility $\mathrm{Ag}-\mathrm{Cu}$ nanoparticles and their thermal transport properties., Metallurgical and Materials Transactions A., 2006; 37: 2033.

15. Junggwon $Y$, Kyoungah C, Byoungjun $\mathrm{P}$, HoChul K, Byeong KL, Sangsig KJ., J Appl Phys., 2008; 47: 5070.

16. Mohl M, Dobo D, Kukovecz A, Konya Z, Kordas K, Wei J, Vajtai R, Ajayan PM.,Electrocatalytic Properties of Carbon Nanotubes Decorated with Copper and Bimetallic CuPd Nanoparticles., J Phys Chem C., 2011; 115: 9403 . into promising deliverable substances.

17. Fan TX, Chow SK, Zhang D., Biomorphic mineralization: from biology to materials., Progress in Materials Sci., 2009; 54(5): 542659.

18. Song CX, Labhasetwar V, Murphy H, Qu X, Humphrey WR, Shebuski RJ, Levy RJ., Formulation and characterization of biodegradables nanoparticles for intravascular local drug delivery., J Control Release., 1997; 43:197- 212.

19. Jaiswal J, Gupta SK, Kreuter J., Preparation of biodegradable cyclosporine nanoparticles by high-pressure emulsification solvent evaporation process., J Control Release., 2004; 96:1692-1778.

20. Soppinath KS, Aminabhavi TM, Kulkurni AR, Rudzinski WE., Biodegradable polymeric nanoparticles as drug delivery devices., J Control Release., 2001; 70:1-20.

21. Tice TR, Gilley RM., Preparation of injectable controlledrelease microcapsules by solventevaporation process., J Control Release., 1985; 2:343-352.

22. Koosha F, Muller RH, Davis SS, Davies MC., The surface chemical structure of poly (hydroxybutyrate) microparticles produced by solvent evaporation process., J Control Release., 1989; 9:149-57.

23. Lemarchand C, Gref R, Passirani C, Garcion E, Petri B, Muller R., Influence of polysaccharide coating on the interactions of nanoparticles with biological systems., Biomaterials., 2006; 27:108-18.

24. Tabata J, Ikada Y., Protein pre-coating of polylactide microspheres containing a lipophilic immune potentiator for enhancement of macrophage phagocytosis and activation., Pharm Res., 1989; 6:296-301.

25. Allemann E, Gurny R, Doekler E., Drug-loaded nanoparticlespreparation methods and drug targeting issues., Eur J Pharm Biopharm., 1993; 39:173-91.

26. Bodmeier R, Chen H., Indomethacin polymeric nanosuspensions prepared by microfluidization., J Control Release., 1990; 12:22333.

27. Vandervoort J, Ludwig A., Biodegradable stabilizers in the preparation of PLGA nano particles: a factorial design study., Int J Pharm., 2002; 238:77-92.

28. Ubrich N, Bouillot P, Pellerin C, Hoffman M, Maincent P., Preparation and characterization of propanolol hydrochloride nano particles : A comparative study., J Control release., 2004:291-300.

29. Catarina PR, Ronald JN, Antonio JR., Nano capsulation: Method of preparation of drug loaded polymeric nanoparticles., Nanotech Bio med., 2006; 2:8-21. 
30. Quintanar-Guerrero D, Allemann E, Fessi H, Doelker E., Preparation techniques and mechanism of formation of biodegradable nanoparticles from preformed polymers., Drug Dev Ind Pharm., 1998; 24:1113-28.

31. Jung T, Kamm W, Breitenbach A, Kaiserling E, Xiao JK, Kissel T., Biodegradable nano particles for oral delivery of peptides: is there a role for polymer to affect mucosal uptake., Eur J Pharm Biopharm., 2000; 50:147-60.

32. Takeuchi H, Yamamoto Y., Mucoadhesive nanoparticulate system for peptide drug delivery., Adv Drug Del Rev., 2001; 47: 39-54.

33. El-shabouri MH., Positively charged nano particles for improving the oral bioavailability of cyclosporine-A., Int J Pharm., 2002; 249:1018.

34. Vargas A, Pegaz B, Devefve E, KonanKouakou Y, Lange N, Ballini JP., Improved photodynamic activity of porphyrin loaded into nano particles: an in vivo evaluation using chick embryos., Int J Pharm., 2004; 286: 131- 45.

35. Yoo HS, Oh JE, Lee KH, Park TG., Biodegradable nanoparticles containing PLGA conjugates for sustained release., Pharm Res., 1999; 16: 1114-8.

36. Fessi H, Puisieux F, Devissaguet JP, Ammoury $\mathrm{N}$, Benita S., Nano capsule formation by interfacial deposition following solvent displacement., Int J Pharm., 1989; 55:R1-R4.

37. Chorney M, DAneuberg H, Golomb G., Lipophilic drug loaded nanospheres by nano precipitation: effect of the formulation variables on size, drug recovery and release kinetics., $\mathrm{J}$ Control release., 2002; 83: 389- 400.

38. Puglisi G, Fresta M, Giammona G, Ventura CA., Influence of the preparation conditions on poly(ethylcyanoacrylate) nanocapsule formation., Int J Pharm., 1995; 125: 283-287.

39. Calvo P, Remunan-Lopez C, Vila-Jato JL, Alonso MJ., Novel hydrophilic chitosanpolyethylene oxide nanoprticles as protein carriers., J Appl Polymer Sci., 1997; 63: 125132.

40. Calvo P, Remunan-Lopez C, Vila-Jato JL, Alonso MJ., Chitosan and chitosan/ethylene oxide-propylene oxide block copolymer nanoparticles as novel carriers for proteins and vaccines., Pharm Res., 1997; 14: 1431-1436.

41. Singh SS, Fenniri H, Singh B., Nanotechnologybased drug delivery systems., J Occup Med Toxicol. 2007; 2: 16.

42. McDevitt MR, Chattopadhyay D, Kappel BJ, Jaggi JS, Schiffman SR, Antczak C., Tumor targeting with antibody-functionalized, radiolabeled carbon nanotubes., J Nucl Med. 2007; 48: 1180-9.

43. Zhang Z, Yang X, Zhang Y, Zeng B, Wang S, Zhu T., Delivery of telomerase reverse transcriptase small interfering RNA in complex with positively charged single-walled carbon nanotubes suppresses tumor growth., Clin Cancer Res., 2006; 12: 4933-9.

44. Sinha N, Yeow JT., Carbon nanotubes for biomedical applications., IEEE Trans Nanobioscience., 2005; 4(2): 180-95.

45. Thakral S, Mehta RM., Fullerenes: an introduction and overview of their biological properties.,Ind J Pharm Sci., 2006; 68:13-9.

46. Wim H, Jong D, Paul JA., Drug delivery and nanoparticles: Applications and hazards., Int $\mathrm{J}$ Nanomed., 2008; 3(2): 133-149.

47. Cuimiao Z, Chunxia Li, Shanshan Huang, Zhiyao Hou, Ziyong Cheng, Piaoping Yang, Chong Peng, Jun Lin., Self activated luminescent and mesoporous strontium hydroxyapatite nanorods for drug delivery., Biomaterials.,2010; 31(12): 3374-83.

48. Paula S, Nunes, Ricardo LC, Albuquerque J, Danielle RR, Cavalcante, Marx D, Dantas M, Juliana C, Cardoso, Marília S, Bezerra, Jamille CC, Souza, Mairim R, Serafini, Lucindo J, Quitans J, Leonardo R, Bonjardim, Adriano AS, Araújo., Collagen Based Films Containing Liposome Loaded Usnic Acid as Dressing for Dermal Burn Healing., J Biomed Biotech., 2011; 4(2):981-25.

49. Lieu J, Levine AL, Matton JS, Yamaguchi M, Lee RJ., Nanoparticles as image enhancing agents for ultrasonography., Physics in Medicine and Biology., 2006; 51: 2179-89. 